\title{
Human relaxins (RLNH1, RLNH2), their receptor (RXFP1) and fetoplacental growth
}

\author{
Kelly Yamasato ${ }^{1}$, Pai-Jong Stacy Tsai ${ }^{2}$, James Davis ${ }^{3}$, Sandra Y Yamamoto ${ }^{1}$ and \\ Gillian D Bryant-Greenwood ${ }^{1}$ \\ ${ }^{1}$ Department of Obstetrics, Gynecology and Women's Health, John A. Burns School of Medicine, University of \\ Hawaii, Honolulu, Hawaii, USA, ${ }^{2}$ Department of Obstetrics and Gynecology, Jacobs School of Medicine and \\ Biomedical Sciences, University of Buffalo, Buffalo, New York, USA and ${ }^{3}$ Office of Biostatistics, John A. Burns \\ School of Medicine, University of Hawaii, Honolulu, Hawaii, USA
}

Correspondence should be addressed to K Yamasato; Email: kyamasat@hawaii.edu

\begin{abstract}
Relaxin, a systemic and placental hormone, has potential roles in fetoplacental growth. Human placenta expresses two RLN genes, RLNH1 and $R L N H 2$. Maternal obesity is common and is associated with abnormal fetal growth. Our aims were to relate systemic and cord blood RLNH2, placental RLNs and their receptor (RXFP1) with fetoplacental growth in context of maternal body mass index, and associations with insulin-like growth factor 2 (IGF2) and vascular endothelial growth factor A (VEGFA) in the same placentas. Systemic, cord blood and placental samples were collected prior to term labor, divided by prepregnancy body mass index: underweight/normal $(N=25)$ and overweight/obese $(N=44)$. Blood RLNH2 was measured by ELISA; placental RLNH2, RLNH1, RXFP1, IGF2 and VEGFA were measured by quantitative immunohistochemistry and mRNAs were measured by quantitative reverse transcription PCR. Birthweight increased with systemic RLNH2 only in underweight/normal women $(P=0.036)$. Syncytiotrophoblast RLNH2 was increased in overweight/obese patients $(P=0.017)$ and was associated with placental weight in all subjects $(P=0.038)$. RLNH1 had no associations with birthweight or placental weight, but was associated with increased trophoblast and endothelial IGF2 and VEGFA, due to female fetal sex. Thus, while systemic RLNH2 may be involved in birthweight regulation in underweight/normal women, placental RLNH2 in all subjects may be involved in placental weight. A strong association of trophoblast IGF2 with birthweight and placental weight in overweight/obese women suggests its importance. However, an association of only RLNH1 with placental IGF2 and VEGFA was dependent upon female fetal sex. These results suggest that both systemic and placental RLNs may be associated with fetoplacental growth.

Reproduction (2017) 154 67-77
\end{abstract}

\section{Introduction}

Fetal growth is an important predictor of perinatal morbidity and mortality as well as adultonset cardiometabolic disease (Barker 2006, Unterscheider et al. 2014, Smith et al. 2016). Despite its importance, the physiology underlying normal fetal growth remains incompletely understood. Maternal obesity is associated with both excessive and restricted fetal growth (Higgins et al. 2011, Magann et al. 2013). Thus, the need to understand how fetal growth is controlled is escalating as rates of maternal obesity continue to increase in the United States (Fisher et al. 2013). Increased maternal body mass index (BMI) in the early second trimester is also associated with increased placental weight (Roland et al. 2014), and placental weight itself is independently associated with adultonset disease (Barker et al. 2010).
Relaxin (RLN) is known for its role in remodeling of reproductive tissues (Hisaw 1926) and as a growth factor (McMurtry et al. 1980, Millar et al. 2003). Three RLN genes, RLN1, RLN2 and RLN3 are present in humans and great apes, whereas RLN in other monkeys and mammals have only a single gene analogous to human RLN2 (Hayes 2004). RLN1 and RLN2 are expressed in human decidua, placenta and prostate (Hansell et al. 1991), and RLN3 is primarily expressed in the nervous system of rodents and macaques (Ma et al. 2009). Maternal systemic RLN is relaxin $\mathrm{H} 2(\mathrm{RLNH} 2)$ and secreted from the corpus luteum during gestation, as shown by concurrent ovarian vein and peripheral blood sampling in pregnant women (Weiss et al. 1976). The placenta does not contribute to this circulating RLN, as women with ovum-donated pregnancies, where there is no functional corpus luteum, have undetectable systemic RLN (Johnson et al. 1991). The RLN receptor was first identified as a leucine-rich repeat G-protein-coupled 
receptor (LGR7) (Hsu et al. 2002) currently known as the RLN family peptide receptor 1 (RXFP1) and is expressed in placenta (Lowndes et al. 2006). Although the function of RLNH1 remains unknown (Bathgate et al. 2013), it shares the same receptor as RLNH2, but binds with a fivefold lower affinity than RLNH2, suggesting preferential RLNH2 binding (Garibay-Tupas et al. 1995).

RLN and RXFP1 are expressed by the endothelium and vascular smooth muscle of the systemic vasculature in rodents (Jelinic et al. 2014) and RXFP1 in umbilical endothelium and vascular smooth muscle in humans (Sarwar et al. 2015). Evidence for roles in both vasodilation and angiogenesis have been obtained primarily from animal models, but potential contributions of systemic RLN to human maternal gestational vascular adaptations are likely (Conrad 2011). RLNH1 and RLNH2 as local hormones can potentially target fetal-placental vessels to increase placental perfusion, with important implications for its involvement in fetal growth. However, little is known about the roles of RLN within the placenta and its potential effects on placental vasculature or in placental growth.

Fetal growth may be partially regulated by systemic and/or placental RLN either directly or indirectly by induction of other fetoplacental growth factors such as insulin-like growth factor 2 (IGF2) (Millar et al. 2003) and vascular endothelial growth factor A (VEGFA) (Unemori et al. 1999). Growth-restricted fetuses exhibit decreased placental IGF2 expression (McMinn et al. 2006, Demetriou et al. 2014), and IGF2 dysregulation is associated with Beckwith-Wiedemann syndrome, a fetal overgrowth condition (Shuman et al. 1993-2016). RLNH2 increases transcription of IGF2 in human fetal membranes (Millar et al. 2003). VEGFA promotes vascular endothelial cell growth and vasodilation and contributes to placental angiogenesis (Andraweera et al. 2012), and fetal growth restriction is associated with decreased maternal serum VEGF (Savvidou et al. 2006). RLNH2 has been shown to induce the expression of VEGF in endometrial cells and monocytes (Unemori et al. 1999, 2000). However, little is known about the relationships between RLNH2, RLNH1 and either IGF2 or VEGFA in the human placenta.

A better understanding of fetal growth is integral to the goal of optimizing the intrauterine environment and improving the immediate and lifelong health of the newborn. The aims of this study were to relate maternal and fetal systemic RLNH2 and placental RLNH1, RLNH2 and RXFP1 in the same patients at term and to show whether there were any associations with fetoplacental growth and levels of placental IGF2 or VEGFA in the context of maternal BMI.

\section{Materials and methods}

\section{Study participants and sample collection}

The sample set was used previously (Tsai et al. 2015a,b) and approved by the Western Institutional Review Board with all participants providing written informed consent. Women at greater than or equal to 37 weeks 0 days gestation with a singleton pregnancy were recruited at Kapiolani Medical Center for Women and Children (Honolulu, HI, USA). Potential participants were limited to those undergoing prelabor cesarean delivery to avoid potentially confounding changes related to labor. Patients with premature rupture of membranes, pregestational diabetes, acute infection at the time of delivery, maternal inflammatory/connective tissue/ autoimmune disease and steroid use were excluded. In addition, patients with gestational diabetes were excluded due to its association with increased fetal growth (Langer et al. 2005). A placental pathologist examined all tissues, and those with histologic signs of inflammation/infection were discarded. Patients were divided by prepregnancy body mass index (BMI) into underweight/normal (Uw/N) (BMI: $16.0-24.9 \mathrm{~kg} / \mathrm{m}^{2}$, $n=25)$ and overweight/obese (Ow/Ob) (BMI $\geq 25.0 \mathrm{~kg} / \mathrm{m}^{2}$, $n=44$ ). Fasting maternal blood was collected prior to delivery and venous cord blood within 15 min of delivery. Plasma was aliquoted and stored at $-80^{\circ} \mathrm{C}$. Two full-thickness placental samples, excluding basal plate, were collected at the cord insertion site within $15 \mathrm{~min}$ of delivery. One sample was fixed in neutral-buffered formaldehyde for $72 \mathrm{~h}$, and the other was immediately stored at $-80^{\circ} \mathrm{C}$.

\section{Maternal plasma analysis}

Maternal plasma interleukin-6 (IL-6) is increased in obesity (Eder et al. 2009) and was measured by High-Sensitivity Quantikine ELISA (R\&D Systems), sensitivity $0.016 \mathrm{pg} / \mathrm{mL}$ and intra-assay coefficient of variation 7.8\%. Maternal and fetal plasma RLN were measured by ELISA (Alpco, Salem, $\mathrm{NH}$, USA), sensitivity $4 \mathrm{pg} / \mathrm{mL}$ and intra-assay coefficient of variation $5.2 \%$. This ELISA was not reported to be specific for RLNH2, but prior work has demonstrated that only RLNH2 is systemic (Johnson et al. 1991). Maternal plasma samples were unavailable for all subjects; therefore, for RLNH2, Uw/N $(n=24)$ and $\mathrm{Ow} / \mathrm{Ob}(n=43)$ and for IL-6, Uw/N $(n=22)$ and $\mathrm{Ow} / \mathrm{Ob}(n=39)$ were used. All samples were run in duplicate.

\section{Placental immunohistochemistry and quantitation}

Immunohistochemistry was performed for quantitative assessment (Taylor \& Levenson 2006). Serial sections $(5 \mu \mathrm{m})$ were heated at $95^{\circ} \mathrm{C}$ for $30 \mathrm{~min}$ in sodium citrate buffer $(10 \mathrm{mM}, \mathrm{pH} 6.0)$ for antigen retrieval for all antigens except VEGFA, which was heated in $10 \mathrm{mM}$ Tris/1 mM EDTA, pH 9.0, for $20 \mathrm{~min}$. The Vectastain Elite kit (Vector Labs, Burlingame, CA, USA) was used according to the manufacturer's protocol. Nonspecific binding was blocked with normal goat serum for $30 \mathrm{~min}$ prior to incubation for $60 \mathrm{~min}$ with either rabbit monoclonal to RLNH2 (Abcam ab 183505, $0.06 \mu \mathrm{g} / \mathrm{mL}$ ), mouse monoclonals to RLNH1 from both Abcam, (ab 14329) and R\&D Systems (MAB 3257, $3 \mu \mathrm{g} / \mathrm{mL}$ ), RXFP1 (SigmaAldrich, $0.06 \mu \mathrm{g} / \mathrm{mL}$ ), and IGF2 (Novus Biologicals, Littleton, CO, USA, $0.5 \mu \mathrm{g} / \mathrm{mL}$ ) or rabbit polyclonal to VEGFA (Santa Cruz Biotechnology, $0.4 \mu \mathrm{g} / \mathrm{mL}$ ). Negative controls were species-specific non-immune $\lg$ at equivalent concentrations with 3,3-diaminobenzidine substrate for visualization. 
Slides were rinsed, counterstained with hematoxylin, cleared and mounted.

Imaging was performed with an Olympus BX51 microscope (Olympus America) and a CRI Nuance spectral analyzer (Caliper Life Sciences, Hopkinton, MA, USA). A series of brightfield images were acquired between 420 and $700 \mathrm{~nm}$ wavelengths at 20-nm intervals. These images were stacked to create a 3-dimensional image cube. The Inform software (version 2.0.2; PerkinElmer) segmented tissues into cell types, unmixed the spectral components and quantitated staining levels (Stack et al. 2014). Average signal intensity per pixel from 5 different fields per patient was obtained as mean optical density units (ODU). Signal intensities were quantitated at 400× magnification. Quantitation of villous vascular endothelium was by manual identification of vessels on each image. Laboratory personnel were blinded to clinical information.

\section{$R N A$ isolation and quantitative reverse transcription PCR (qRT-PCR)}

Total RNA was extracted from $30 \mathrm{mg}$ placental tissue using the RNeasy Fibrous MiniKit (QIAGEN) and quality was assessed by Agilent Bioanalyzer 2100 (Agilent Technologies). Total RNA (400 ng) was reverse transcribed using Multiscribe reverse transcriptase (Applied Biosystems), incubating at $25^{\circ} \mathrm{C}$ for $10 \mathrm{~min}, 37^{\circ} \mathrm{C}$ for $120 \mathrm{~min}$ and $95^{\circ} \mathrm{C}$ for $5 \mathrm{~min}$. For RT-PCR, gene-specific TaqMan Assays on Demand probe sets for RLN2 (Hs00366417_m1), RLN1 (Hs00762019_s1) and two reference genes SDHA (Hs00188166_m1) and YWHAZ (Hs00237047_ $\mathrm{m} 1$ ) were selected for placenta as recommended (Meller et al. 2005: 1). Reactions in triplicate were cycle at $50^{\circ} \mathrm{C}$ for $2 \mathrm{~min}$, 1 cycle at $95^{\circ} \mathrm{C}$ for $10 \mathrm{~min}, 40$ cycles at $95^{\circ} \mathrm{C}$ for $15 \mathrm{~s}$ and $60^{\circ} \mathrm{C}$ for $1 \mathrm{~min}$. Results were normalized to the geometric mean of SDHA and YWHAZ expression in each sample and reported as the mean \pm S.D.

\section{Statistical analyses}

Data were analyzed with SAS statistical software, version 9.3 (SAS Institute Inc, Cary, NC) and SPSS statistics for Macintosh, version 23 (IBM). Demographic and clinical data were compared by Student's $t$-test and chi-square or
Fisher's exact test for continuous and categorical variables respectively. Immunostaining intensities between Uw/N and $\mathrm{Ow} / \mathrm{Ob}$ patients were compared with Student's t-tests. Relationships between RLNH1 and RLNH2, RXFP1, IGF2, VEGFA, with fetal and placental growth and fetal sex were compared for all samples and stratified by BMI group using linear regression models. $P$ values of $\leq 0.05$ were considered statistically significant.

\section{Results}

\section{Demographic and clinical data}

Of 84 patients, 6 were excluded for chorioamnionitis or placental histologic inflammation and 9 for gestational diabetes, leaving 69 patients in this study. Demographic and clinical data are shown in Table 1. Of these, 3 were underweight (Uw), 22 normal weight $(N), 21$ overweight (Ow) and 23 obese (Ob), which were combined into 2 groups Uw/N $(n=25)$ and Ow/Ob $(n=44)$ with mean pregestational BMls of 21.3 and 32.0 respectively $(P<0.001)$. The Uw/N group was significantly older than the Ow/Ob group $(P=0.034)$, but otherwise there were no differences. Maternal plasma IL-6 and placental weight in relation to prepregnancy $\mathrm{BMI}$ are shown in Fig. $1 \mathrm{~A}$ and $\mathrm{B}$ respectively. Maternal IL-6 $(P<0.001)$ (Fig. 1A) and placental weight $(P=0.025)$ (Fig. 1B) both increased linearly with prepregnancy BMI, validating our sample set as these observations were consistent with previous data (Eder et al. 2009, Roland et al. 2014).

\section{Maternal and cord plasma RLNH2}

Birthweight increased as maternal plasma RLNH2 increased for $U \mathrm{w} / \mathrm{N}(P=0.036)$ but not for $\mathrm{Ow} / \mathrm{Ob}$ patients $(P=0.553)$ (Fig. 1C). There was no association between maternal plasma RLNH2 and placental weight (data not shown). The mean \pm S.D. of maternal and cord plasma RLNH2 levels for all patients were $260 \pm 158.8 \mathrm{pg} /$ $\mathrm{mL}$ and $22 \pm 12.5 \mathrm{pg} / \mathrm{mL}$ respectively, with cord plasma RLNH2 significantly lower than maternal RLNH2 $(P<0.001)$. Data for all patients showed cord plasma

Table 1 Demographic and clinical characteristics of underweight/normal and overweight/obese patients.

\begin{tabular}{|c|c|c|c|}
\hline Characteristics & $\mathbf{U w} / \mathbf{N}(n=25)$ & Ow/Ob $(n=44)$ & Significance $(P)$ \\
\hline Maternal age at (years) & $33 \pm 5.9$ & $30 \pm 5.0$ & $0.034^{*}$ \\
\hline Primiparous & $6(24.0)$ & $7(15.9)$ & 0.524 \\
\hline Pregestational BMI (kg/m²) & $21.3 \pm 2.2$ & $32.0 \pm 6.3$ & $<0.001^{*}$ \\
\hline Male newborn & $8(32.0)$ & $25(56.8)$ & 0.083 \\
\hline Gestational age at delivery (weeks) & $38.9 \pm 0.7$ & $39.0 \pm 0.5$ & 0.237 \\
\hline Gestational weight gain (pounds) & $13.2 \pm 5.13$ & $14.9 \pm 7.5$ & 0.570 \\
\hline Smoking at delivery & $0(\overline{0.0})$ & $3(6.8)$ & 0.160 \\
\hline Hypertension & $0(0.0)$ & $2(4.5)$ & 0.531 \\
\hline Birthweight (g) & $3259 \pm 418$ & $3445 \pm 483$ & 0.096 \\
\hline Placental weight (g) & $475 \pm 92$ & $535 \pm 102$ & $0.011 *$ \\
\hline
\end{tabular}

Results are expressed as mean \pm S.D. or frequency (\%).

$* P<0.05$.

$\mathrm{Ow} / \mathrm{Ob}$, overweight/obese; Uw/N, underweight/normal. 

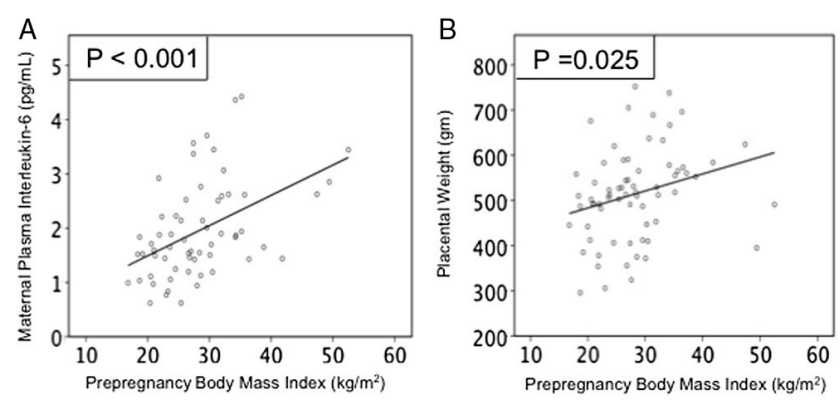

C

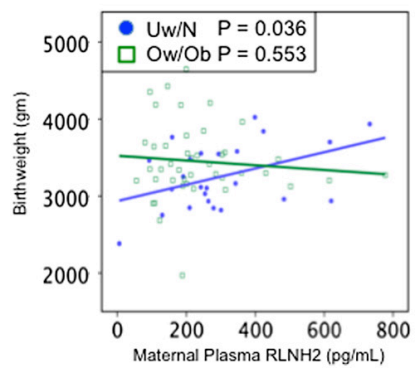

$\mathrm{D}$

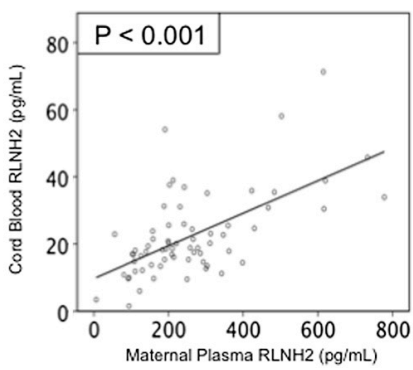

Figure 1 Baseline maternal BMI associations and systemic RLNH2. (A) Maternal plasma IL-6 $(n=61 ; P<0.001)$ and (B) placental weight ( $n=69 ; P=0.025$ ) both increased as maternal prepregnancy BMI increased. (C) Birthweight increased as maternal plasma RLN2 increased in Uw/N (closed circles) $(n=24 ; P=0.036)$, but not Ow/Ob (open squares) $(n=43 ; P=0.553)$. (D) Cord blood RLNH2 increased as maternal plasma RLNH2 increased $(n=67 ; P<0.001)$.

levels increased as maternal plasma RLNH2 increased $(P<0.001$, Fig. 1D), but there were no associations of cord plasma RLNH2 with either birthweight or placental weight. From these data, the levels of maternal systemic RLNH2 may be more important for birthweight in $U w / N$ than those in $\mathrm{Ow} / \mathrm{Ob}$ women.

\section{Placental RLNH2: protein and gene expression}

In the placenta, RLNH2 immunolocalized strongly to the syncytiotrophoblast and the few remaining cytotrophoblasts, with lighter staining in the fetal vascular endothelial cells. Immunostaining was also lighter in Uw/N compared to Ow/Ob samples, examples shown (Fig. 2A and B) with a negative control (Fig. 2C). Quantitation with all subjects showed that placental weight increased as syncytiotrophoblast $\mathrm{RLNH} 2$ increased $(P=0.038)$ (Fig. 2D), with no increase in placental weight with RLNH2 in the vasculature. However, there were no relationships between syncytiotrophoblast $(P=0.185)$ or vascular $(P=0.942)$ RLNH2 and birthweight. Subdivision of patients into two groups, showed that syncytiotrophoblast RLNH2 was significantly greater in $\mathrm{Ow} / \mathrm{Ob}$ patients compared to $\mathrm{Uw} / \mathrm{N}$ patients $(P=0.017)$ (Fig. $2 \mathrm{E}$ and $\mathrm{F})$. In contrast, for the two groups of patients, there was no significant difference in $\mathrm{RLNH} 2$ in the placental vasculature (not shown). Placental RLNH2 mRNA in Ow/Ob patients
(Fig. 2G) showed a similar pattern with placental weight as its protein (Fig. 2F), as well as with birthweight (not shown), neither were significant. However, the RLNH2 mRNA for Uw/N subjects differed from that for Ow/ Ob subjects and was significantly but inversely related to both placental weight $(P=0.018)$ (Fig. 2G) and birthweight ( $P=0.017)$ (not shown).

\section{Placental RXFP1}

The relaxin receptor (RXFP1) was immunolocalized in the same placentas and examples are shown for Uw/N (Fig. 3A) and $\mathrm{Ow} / \mathrm{Ob}$ (Fig. 3B) with a negative control (Fig. 3C). The staining intensity was very good in the syncytiotrophoblast and vascular endothelial cells, with additional light and intermittent staining in the vascular smooth muscle as previously shown (Jelinic et al. 2014). Separate quantitation of these was not possible; therefore, the results for the vasculature were a combination of both endothelial and smooth muscle staining. Quantitation showed significantly more RXFP1 in the syncytiotrophoblast of Ow/Ob compared to the Uw/N patients $(P=0.054)$ (Fig. 3D) with no difference in the vasculature $(P=0.664)$ (Fig. $3 \mathrm{E})$. When plotted for individual subjects, placental weight was not associated with RXFP1 in either the syncytiotrophoblast $(P=0.977)$ (Fig. 3F) or vasculature ( $P=0.093)$ (Fig. 3G).

\section{Summary: maternal and placental RLNH2 and RXFP1, according to prepregnancy maternal BMI}

A diagram summarizing these data is shown in Fig. 4. Maternal systemic RLNH2, a product of the corpus luteum, was increased as birthweight increased in Uw/N patients only. There was no association with placental weight. However, for all subjects, syncytiotrophoblast RLNH2 was related to placental weight, as shown. In the $\mathrm{Ow} / \mathrm{Ob}$ patients, who had heavier placentas, there were also relatively higher levels of syncytiotrophoblast RLNH2 and RXFP1 compared to the Uw/N patients. These data suggest that in $U w / N$ patients, maternal plasma RLNH2 may be associated with birthweight, whereas placental $\mathrm{RLNH} 2$ in all subjects may be associated with placental weight.

\section{Placental RLNH2 and associations with IGF2 and VEGFA}

RLNH2 has been shown to increase IGF2 (Millar et al. 2003) and VEGFA (Unemori et al. 1999) in different cell types. Although any direct relationship has not been shown in placental tissue or cells, we sought associations between RLNH2 and these important placental growth factors. Therefore, we immunolocalized both proteins in the placentas of our patients and sought associations with the concomitant expression of RLNH2. IGF2 localized to the remaining cytotrophoblast and vascular 
A

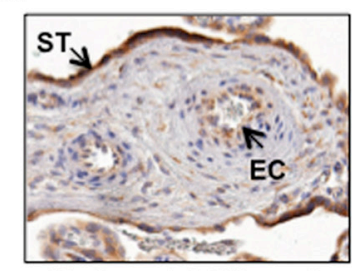

Underweight/Normal
B

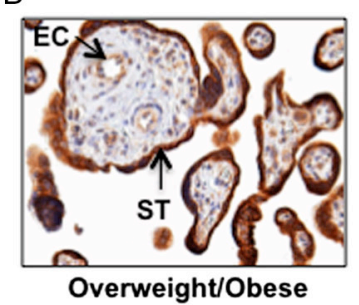

C

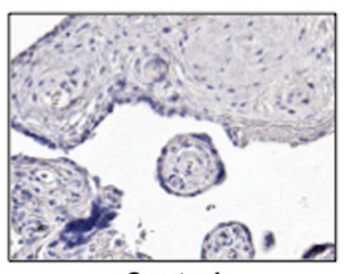

Control
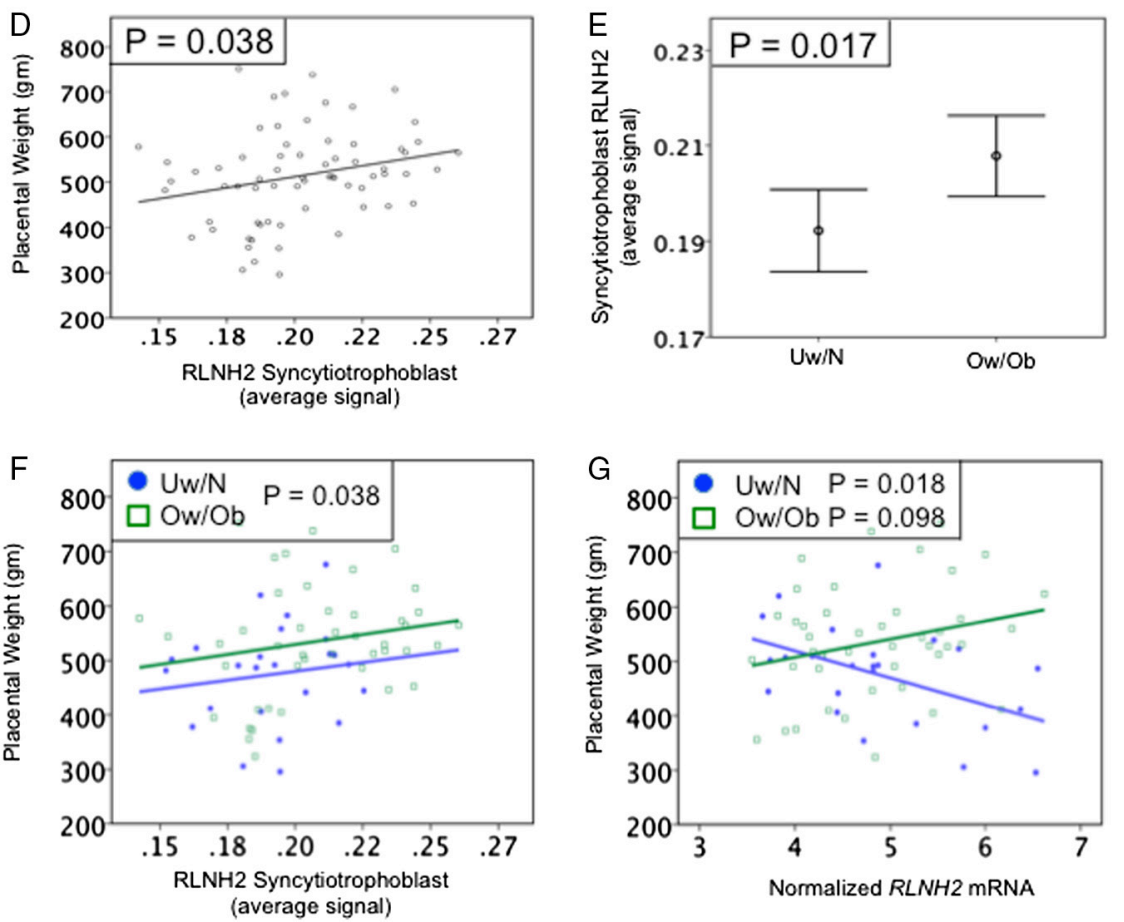

Figure 2 Placental RLNH2 and fetoplacental growth. Examples of placental RLNH2 immunostaining in (A) Uw/N, (B) Ow/Ob and (C) negative control. RLNH2 immunostained the syncytiotrophoblast (ST) and villous vascular endothelial cells (EC). (D) Quantitation for all subjects $(n=69)$ showed that placental weight increased as syncytiotrophoblast RLNH2 increased $(P=0.038)$. (E) Syncytiotrophoblast RLNH2 also increased in Ow/Ob compared to Uw/N subjects $(P=0.017)$. (F) Plotted for individual subjects, syncytiotrophoblast RLNH2 increased with placental weight for both Uw/N (closed circles) $(n=25)$ and Ow/Ob (open squares) $(n=44)$. (G) Placental weight was inversely related to placental $R L N H 2$ mRNA for Uw/N (closed circles) $(P=0.018)$, but not $\mathrm{Ow} / \mathrm{Ob}$ (open squares) $(P=0.098)$. Original magnification $400 \times$. endothelium, examples are shown for Uw/N (Fig. 5A) and $\mathrm{Ow} / \mathrm{Ob}$ (Fig. 5B), with a negative control (Fig. 5C). Placental weight $(P<0.001)$ and birthweight $(P=0.002)$ (Fig. 5D and E respectively) both significantly increased as cytotrophoblast IGF2 increased in the $\mathrm{Ow} / \mathrm{Ob}$ patients, but not in the Uw/N patients.

VEGFA immunolocalized to the syncytiotrophoblast and remaining cytotrophoblast, with slightly stronger staining in the latter. It also immunolocalized to the vascular endothelium. These results agree with results from Clark and coworkers (1996) and therefore are not shown here. There were no significant associations between placental RLNH2 and either IGF2 or VEGFA in the trophoblast or placental vasculature.

\section{Placental RLNH1 and associations with RXFP1, IGF2 and VEGFA}

The negative results showing lack of any association of RLNH2 with the growth factors, IGF2 and VEGFA were unexpected. We therefore considered the homologous hormone RLNH1, also expressed in the placenta (Hansell et al. 1991). The recent availability of antibodies to RLNH1 allowed us explore this in our samples. Initially, we used an antibody from the same source as the RLNH2 antibody used here, but this was unsuitable for immunohistochemistry in the placenta, giving high background staining. We therefore used another recently available antibody to RLNH1 (see 'Materials and methods' section). This showed specific localization to the syncytiotrophoblast, remaining cytotrophoblast and fetal endothelium, examples are shown for Uw/N (Fig. 6A) and Ow/Ob (Fig. 6B), with a negative control (Fig. 6C). There were no differences in staining intensity in the two patient groups. Quantitation of RLNH1 according to placental weight showed different results from those of RLNH2, with no significant changes in the groups of patients, although the levels in $\mathrm{Ow} / \mathrm{Ob}$ patients diverged from those of the Uw/N group (Fig. 6D). However, levels of mRNA for RLNH1 according to placental weight (Fig. 6E) showed no decline in the Uw/N patients as did mRNA for RLNH2 (Fig. 2G); indeed, placental weight tended to increase as RLNH1 mRNA increased $(P=0.07)$.

The associations of placental RLNH1 and RLNH2 proteins with placental expression of IGF2 and VEGFA 
A

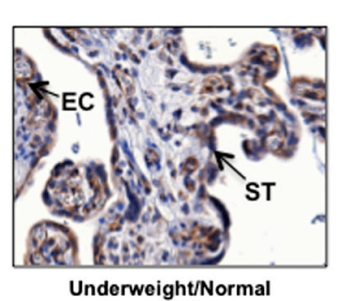

B

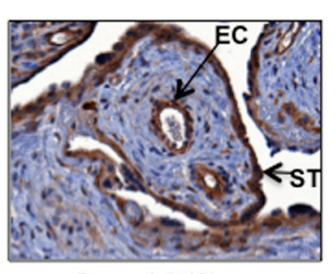

Overweight/obese

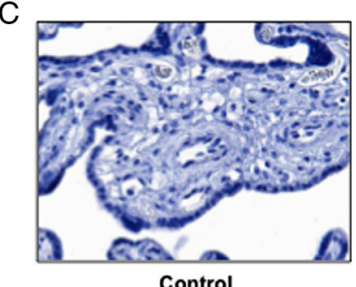

Control

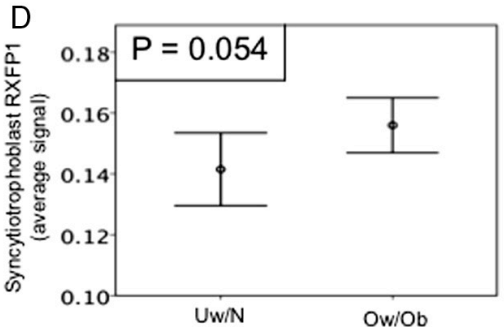

E
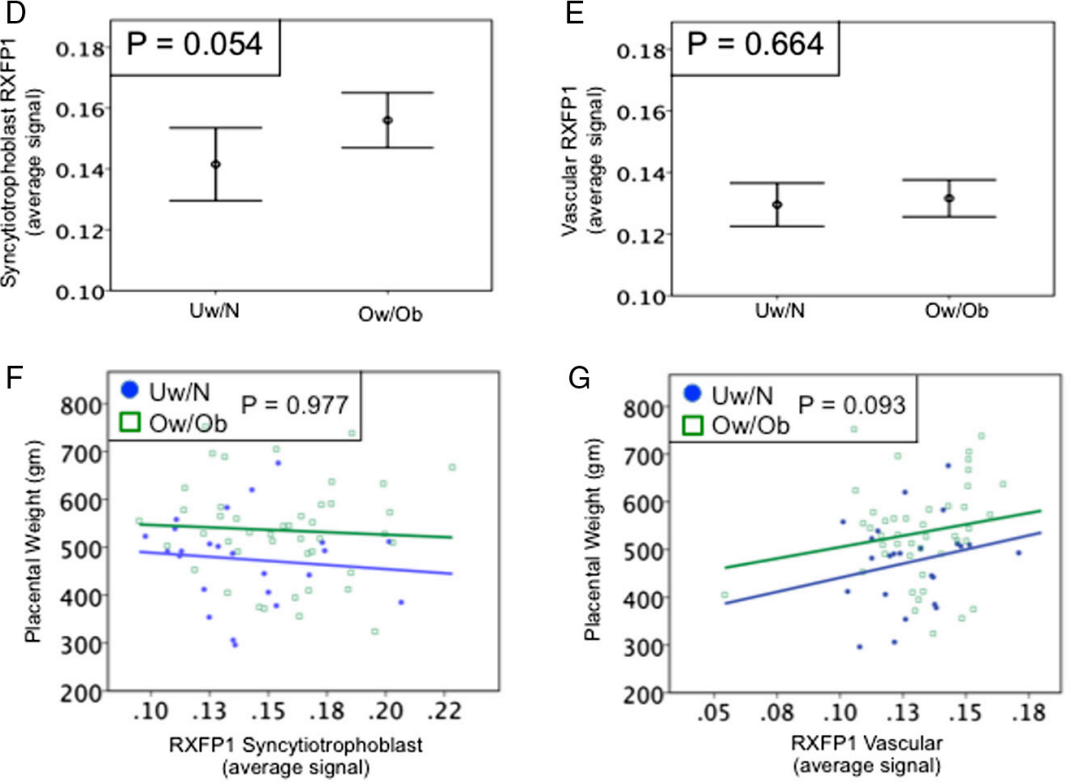

Figure 3 Placental RXFP1 and placental weight. Examples of placental immunostaining in (A) Uw/N, (B) Ow/Ob and (C) negative control. RXFP1 immunostained the syncytiotrophoblast (ST) and villous vascular endothelial cells (EC), with light and intermittent staining in the vascular smooth muscle. (D) Quantitation showed RXFP1 was increased in the syncytiotrophoblast of Ow/Ob compared to Uw/N subjects $(P=0.054)$, (E) with no difference in the fetal vasculature $(P=0.664)$. (F) Placental weight was not associated with syncytiotrophoblast RXFP1 for either Uw/N or Ow/Ob $(P=0.977$, all subjects). (G) For all subjects, there was a trend for placental weight to increase with vascular RXFP1 $(P=0.093)$. Original magnification $400 \times$.

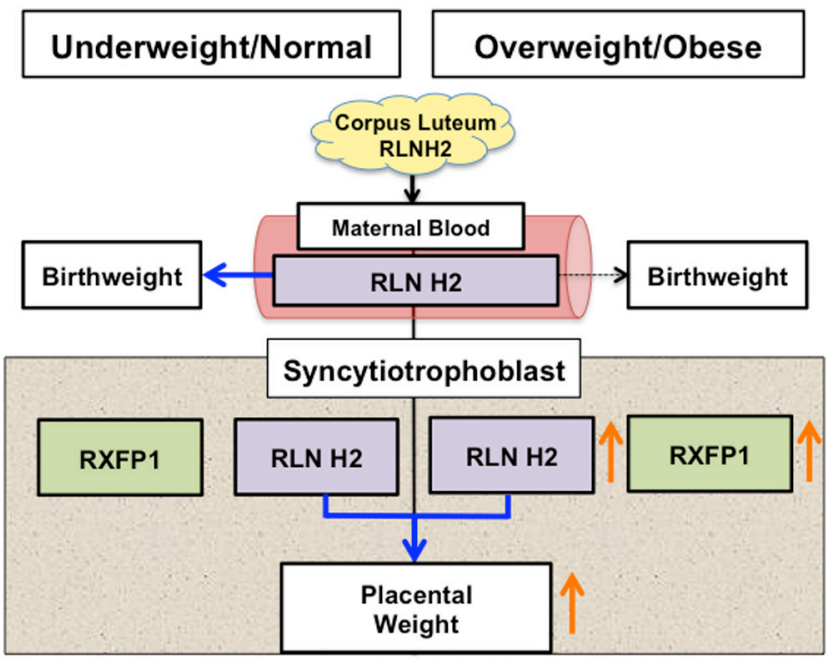

Figure 4 Summary of BMI-dependent associations between maternal and syncytiotrophoblast RLNH2 and fetoplacental growth, with $\mathrm{Uw} / \mathrm{N}$ subjects on the left and $\mathrm{Ow} / \mathrm{Ob}$ on the right of the figure. Solid arrows show significant positive associations and dashed arrows non-significant associations. Birthweight increased as maternal plasma RLNH2 increased for Uw/N $(P=0.036)$ but not $\mathrm{Ow} / \mathrm{Ob}(P=0.553)$ subjects. However, for all subjects, placental weight increased as syncytiotrophoblast RLNH2 increased $(P=0.038)$, and syncytiotrophoblast RLNH2 $(P=0.017)$, RXFP1 $(P=0.054)$ and placental weight $(P=0.019)$ all increased in $\mathrm{Ow} / \mathrm{Ob}$ compared to $\mathrm{Uw} / \mathrm{N}$ subjects (orange arrows). are due to their actions on the same receptor (RXFP1) (Garibay-Tupas et al. 1995) as well as the ability of RLN to increase expression of RXFP1. This has been demonstrated in vitro, in isolated decidual cells (Mazella et al. 2004) and in full-thickness fetal membrane explants (Lowndes et al. 2006). In this study, in the syncytiotrophoblast, we found a significant positive association of RLNH2 with syncytiotrophoblast RXFP1 $(P<0.001)$, and of RLNH1 with RXFP1 $(P=0.005)$ in the same tissues. Similarly, within the vascular endothelium, RLNH2 $(P=0.009)$ and RLNH1 $(P=0.058)$ were positively associated with RXFP1. Thus, both RLNH1 and RLNH2 were likely to have positively affected the expression of RXFP1.

In spite of this, there was a markedly different association of RLNH1 and RLNH2 with IGF2 and VEGFA in the trophoblast and vasculature (Table 2). This shows a highly significant association of RLNH1 with IGF2 (trophoblast $P<0.001$, vasculature $P=0.002$ ) and VEGFA (trophoblast $P=0.001$, vasculature $P=0.043$ ), and no significant associations for RLNH2 with either IGF2 or VEGFA.

Although syncytiotrophoblast RLNH1 levels showed no differences between women with male or female fetuses $(P=0.537)$, its associations with IGF2 and VEGFA differed significantly by fetal sex (Table 3 ). IGF2 in the trophoblast increased as trophoblast RLNH1 increased in female $(P<0.001)$ but not in male $(P=0.6)$ fetuses. 
A

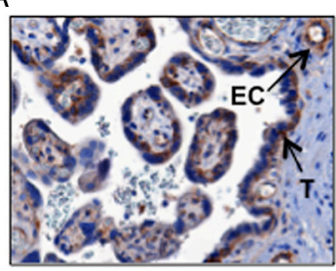

Underweight/Normal
B

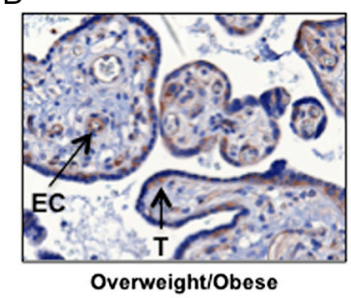

C

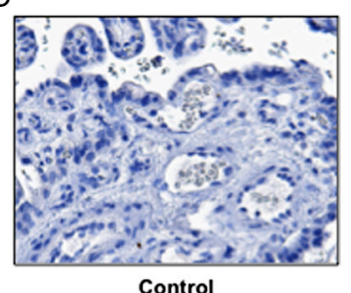

Control
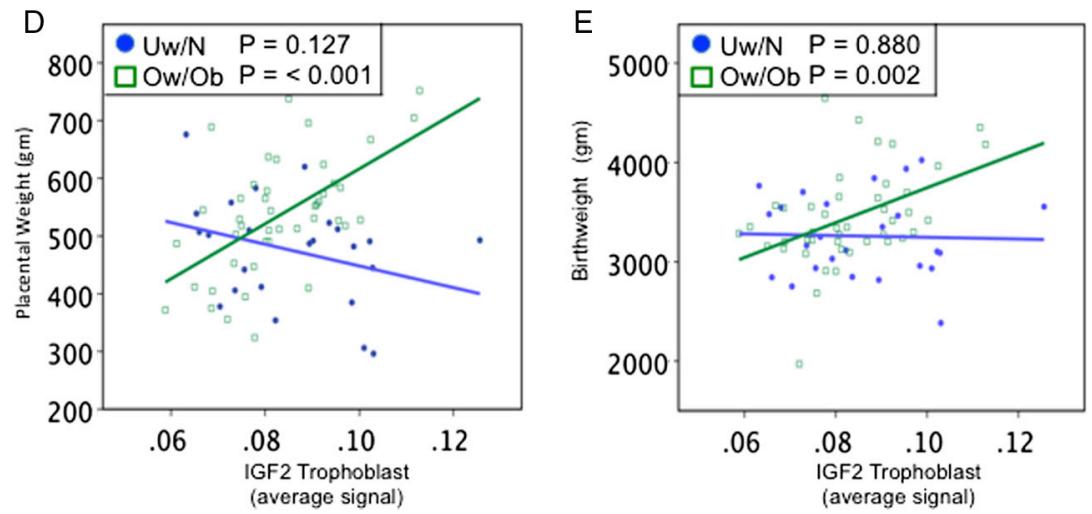

Figure 5 Placental IGF2 and fetoplacental growth. Examples of IGF2 immunostaining in (A) Uw/N, (B) Ow/Ob and (C) negative control. Anti-IGF2 only immunostained the remaining cytotrophoblast $(\mathrm{T})$ and villous vascular endothelial cells (EC). (D) Quantitation showed that placental weight increased as IGF2 increased for $\mathrm{Ow} / \mathrm{Ob}$ $(P<0.001)$ (open squares) but not Uw/N subjects (closed circles). (E) Birthweight increased as IGF2 increased for $\mathrm{Ow} / \mathrm{Ob}$ subjects ( $P=0.002$ ) (open squares) and not Uw/N subjects (closed circles).
Similarly, vascular IGF2 increased as trophoblast RLNH1 increased for female $(P=0.005)$, but not for male fetuses $(P=0.4)$. Trophoblast VEGFA was also more closely associated with trophoblast RLNH1 in female $(P=0.002)$ compared to male fetuses $(P=0.3)$, whereas vascular VEGFA was also significantly associated with RLNH1 in female $(P<0.001)$ but not male fetuses $(P=0.1)$.

\section{Discussion}

This study is the first to suggest the possible roles for maternal systemic RLNH2 as well as placental
RLNH2 and RLNH1 in fetoplacental growth. We have shown associations at term between maternal plasma $\mathrm{RLNH} 2$ with birthweight, syncytiotrophoblast RLNH2 with placental weight and RLNH1 with placental IGF2 and VEGFA in women with a female fetus. In addition, the relationship between maternal systemic RLNH2 and cord plasma levels suggests the possible transfer of RLNH2 from maternal blood. We have also demonstrated strong immunostaining of RXFP1 in the endothelium of placental vessels, suggesting that RLNs may act as vasodilators. Thus, these results are consistent with potential roles for RLNs in increasing placental
A

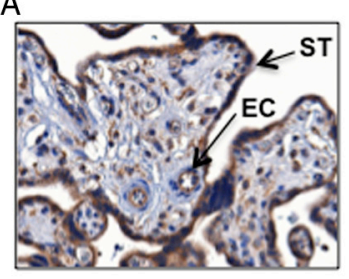

Underweight/Normal

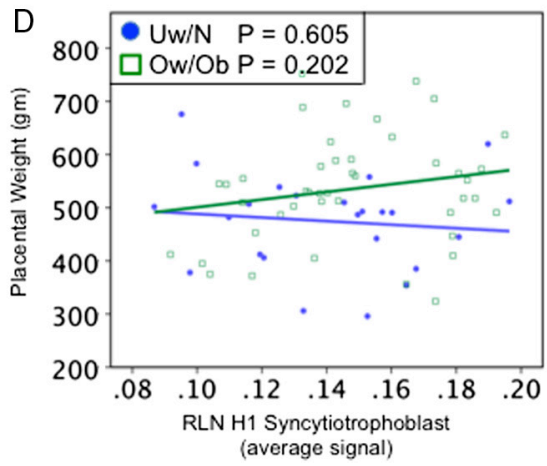

B

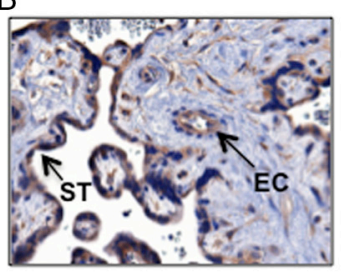

Overweight/Obese

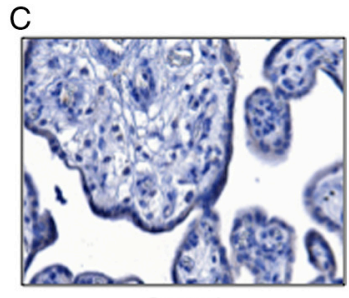

Control

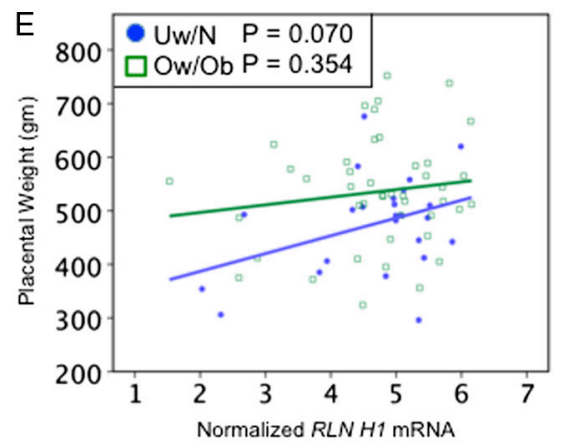

Figure 6 Placental RLNH1 and placental weight. Examples of RLNH1 immunostaining in (A) Uw/N, (B) Ow/Ob and (C) negative control. Anti-RLNH1 immunostained the syncytiotrophoblast (ST) and villous vascular endothelial cells (EC). (D) Quantitation showed that syncytiotrophoblast RLNH1 was unrelated to placental weight for either $U w / N$ $(P=0.605)$ or $\mathrm{Ow} / \mathrm{Ob}(P=0.202)$ patients. (E) Placental RLNH1 mRNA showed a tendency to increase as placental weight increased $(P=0.070)$ in $U \mathrm{w} / \mathrm{N}$ subjects (closed circles) with no relationship in $\mathrm{Ow} / \mathrm{Ob}$ patients $(P=0.354)$ (open squares). 
Table 2 Associations of placental RLNH1 and RLNH2 with placental expression of IGF2 and VEGFA in the trophoblast and vasculature.

\begin{tabular}{llcc}
\hline Tissue & Fetal-placental growth factor & RLNH1 $*$ & RLNH2 \\
\hline \multirow{2}{*}{ Trophoblast } & IGF2 & $<0.001$ & 0.5 \\
& VEGFA & 0.001 & 0.1 \\
\multirow{2}{*}{ Vasculature } & IGF2 & 0.002 & 0.9 \\
& VEGFA & 0.043 & 0.2 \\
\hline
\end{tabular}

Results expressed as $P$-value of association.

* Positive relationship for all associations.

IGF2, insulin-like growth factor 2; RLNH1, relaxin $\mathrm{H} 1$; $\mathrm{RLNH}$ 2, relaxin $\mathrm{H} 2$; VEGFA, vascular endothelial growth factor $\mathrm{A}$.

perfusion, in addition to possibly affecting local growth factor production. Together, these could increase fetoplacental growth.

Maternal systemic RLN is only a product of the corpus luteum (Weiss et al. 1976, Johnson et al. 1991). A progressive relationship between increasing obesity and decreasing reproductive hormone secretion with evidence for decreased corpus luteum function in obese women has suggested that obesity plays a role in relative infertility (Rochester et al. 2009). It is possible that a healthier corpus luteum in the Uw/N subjects resulted in the significant relationship between maternal RLNH2 and birthweight. The absence of any relationship between maternal $\mathrm{RLNH} 2$ and birthweight in the $\mathrm{Ow} / \mathrm{Ob}$ patients is also consistent with previous work showing decreased vascular responsiveness to systemic RLNH2 in overweight non-pregnant rats (van Drongelen et al. 2012). We have also shown no association between maternal RLNH2 and placental weight, suggesting that systemic RLNH2 is probably not involved in the regulation of placental growth, and that this is more likely to be controlled locally within the placenta. Thus, syncytiotrophoblast RLNH2 and RXFP1 were both increased in Ow/Ob women compared to the Uw/N group, which may be related to their heavier placentas. Interestingly, although increasing syncytiotrophoblast RLNH2 was associated with increasing placental weight, regardless of maternal $\mathrm{BMI}$, increasing $\mathrm{RLNH} 2$ mRNA was associated with decreasing birthweight and placental weight in the $U \mathrm{w} / \mathrm{N}$ women, possibly reflecting an acute compensatory effect or negative feedback.

Table 3 Comparisons between associations of RLNH1 and placental IGF2 and VEGFA with fetal sex.

\begin{tabular}{llcc}
\hline & & \multicolumn{2}{c}{ Trophoblast RLNH1 } \\
\cline { 3 - 4 } Tissue & Fetal-placental & & \\
\cline { 3 - 4 } Trophoblast & growth factor & Female fetus* & Male fetus \\
\hline \multirow{2}{*}{ Vasculature } & IGF2 & $<0.001$ & 0.6 \\
& VEGFA & 0.002 & 0.3 \\
& IGF2 & 0.005 & 0.4 \\
& VEGFA & $<0.001$ & 0.8 \\
\hline
\end{tabular}

Results expressed as $P$ value of association.

*Positive relationship for all associations.

IGF2, insulin-like growth factor 2; RLNH1, relaxin H1; VEGFA,

vascular endothelial growth factor $\mathrm{A}$.
For this study, we have used some novel and newly developed commercial reagents for measurement of both RLNH1 and RLNH2 mRNAs and proteins. The high level of homology for the RLNs at both the RNA and protein levels makes specific identification of one in the presence of the other difficult. Over a period of years, we have reported the measurement of RNAs for both hormones using different strategies (Hansell et al. 1991, Garibay-Tupas et al. 1995), as well as raising antisera to synthetic peptides of RLNH1 and RLNH2 (Tashima et al. 2002). While it is normal to report methods of production of reagents, for those commercially available this can be proprietary. However, information provided on the reagents used here stated that the RLNH1 primers and probe mapped within a single exon, but genomic DNA may be detected. We therefore used only highquality RNA devoid of DNA contamination. For the antibodies, we tried to use equivalent reagents for RLNH1 and RLNH2, but this was not possible because the equivalent RLNH1 antibody was unsuitable for immunohistochemistry. Therefore, although our anti$\mathrm{RLNH} 2$ was directed to a sequence in the B-chain, the alternative anti-RLNH1 used, was to human prorelaxin-1. However, this antibody had no cross-reactivity with recombinant human prorelaxin-2. We acknowledge that these differences may be important, but our use for intracellular detection by immunohistochemistry, rather than for solution assays like Western blotting or ELISA, may have also been advantageous. Our results for RLNH1 and $\mathrm{RLNH} 2$ were appropriately similar for such highly homologous molecules, while in some instances, they were markedly different. This lends credence to the view that the reagents used here allowed specific detection of the two intracellular hormones. At the same time, we also recognize the need for confirmation of our results with different reagents and different methodologies.

Unfortunately, it was not possible to confirm our results by Western blotting. The low levels of RLNH1 and RLNH2 expression in the placenta, together with their adsorptive properties preclude this methodology. However, it is now possible to make quantitative immunohistochemistry like an immunoassay carried out on a tissue section, but rigorous multiple precautions need to be taken into account (Taylor \& Levenson 2006, Stack et al. 2014). In addition, quantitation needs to be performed with state-of-the-art software allowing specific intracellular measurements (Fiore et al. 2012). Just as the recent availability of reagents for RLNH1, which for the first time has allowed this hormone to be studied concurrently with RLNH2, similarly, new methodologies are becoming available for the study of low levels of local hormones in tissues such as the placenta.

The two human RLNs, RLNH1 and RLNH2, both signal through RXFP1 by G-protein coupling and adenylate cyclase signaling to increase intracellular cAMP levels (Halls etal. 2009). We have shown here strong associations between the levels of both RLNs and that of RXFP1, with 
RLNH2 having a stronger relationship than RLNH1 in both the syncytiotrophoblast and placental vasculature. A considerable body of work has been directed to understanding the action of RLNH2 on RXFP1, which has been shown to be complex and dependent upon the cell type (Halls et al. 2009). Activation of this receptor by RLNH2 uses G-protein signaling, but also tyrosine kinase as another signaling mechanism (Bartsch et al. 2001). Our results on the associations of RLNH1 and RLNH2 with IGF2 and VEGFA were markedly different, suggesting that different signaling mechanisms may be induced by activation of RXFP1 on the syncytiotrophoblast by these two hormones. Further work is required in order to understand how they differentially regulate these two growth factors.

We have shown that RXFP1 is well expressed in late gestation by both the syncytiotrophoblast and vascular endothelium. Vascular responsiveness to $\mathrm{RLNH} 2$ is associated with RXFP1 expression in the endothelium (Jelinic et al. 2014) involving the endothelin-1/nitric oxide vasodilation pathway (Novak et al. 2006). Our results are therefore consistent with a role for RLN in increasing placental perfusion, and as a consequence, it could affect the fetoplacental growth. At the same time, RLNH2 increases VEGF (Unemori et al. 1999, 2000) thereby increasing angiogenesis and influencing growth. IGF2 is a well-recognized fetoplacental growth factor (Fowden 2003, Kappil et al. 2015). The results presented here are the first to suggest a possible maternal BMI-dependent relationship between placental IGF2 and fetoplacental growth, with stronger effects in Ow/ $\mathrm{Ob}$ women. The IGF axis in cord blood is sensitive to maternal $\mathrm{BMI}$, with $\mathrm{BMI}$-dependent differences in IGF2 regulation (Hoyo et al. 2012) and levels of IGFbinding proteins (Ferraro et al. 2012). Further work is needed to see if altered IGF2 in maternal obesity is related to increased body fat and insulin resistance in the newborns of obese mothers (Catalano et al. 2009). Although RLNH2 has been shown to induce IGF2 in the fetal membranes (Millar et al. 2003), our results showed a stronger association with RLNH1 in the placenta, which was similar for VEGFA. As RLNH1 is limited to primates, its functional role as a placental hormone may be important. Although RLNH1 is not a product of the corpus luteum, it is produced in low levels by the syncytiotrophoblast and is perfectly positioned to target syncytiotrophoblast RXFP1 to increase growth factor production. Although this study is preliminary and we have only shown associations, it would be important in the future to show a direct linkage between RLNH1 binding to RXFP1 in the syncytiotrophoblast and the increased production of IGF2 and VEGFA.

The dogma in the RLN field is that it is of greater importance in female compared to male reproduction. We show here that the relationships between placental RLNH1 and both IGF2 and VEGFA were highly specific to female fetal sex. Human data as well as animal models demonstrate sex-specific fetal growth and metabolic adaptations to environmental insults, with female fetuses generally exhibiting greater responsiveness and resilience than male fetuses (Clifton 2005, GonzalezBulnes et al. 2015, Rosenfeld 2015), with improved neonatal outcomes (Simchen et al. 2014, Wallace et al. 2016). Thus, further evaluation of RLNH1 in pathologic settings in pregnancy could assess its contribution to this well-known disparity.

Our data show associations between the RLNs and fetoplacental growth and fetoplacental growth factors, but do not prove causation. We also recognize the need for these results to be confirmed and extended using other methodologies. In addition, our data are also limited to term gestation, while fetoplacental growth at delivery is the cumulative result of processes spanning the whole pregnancy. Studies in earlier gestation are therefore needed, although it would be difficult to obtain normal human samples. In conclusion, these preliminary results suggest that maternal $\mathrm{RLNH} 2$ and placental RLNH1 and RLNH2 may have maternal $\mathrm{BMI}$ - and fetal sex-dependent associations with human fetoplacental growth.

\section{Declaration of interest}

The authors declare that there is no conflict of interest that could be perceived as prejudicing the impartiality of the research reported.

\section{Funding}

This study was supported by the University of Hawaii, John A Burns School of Medicine, Department of Obstetrics, Gynecology, and Women's Health and Partnerships for Cardiometabolic Disparities in Native and Pacific peoples - Research Core (MD000173-13). Statistical analyses were supported in part by grants from the National Institute on Minority Health and Health Disparities, United States (U54MD007584); and the National Institute of General Medical Sciences (1U54GM104944-01A1, P20GM103466).

\section{Acknowledgements}

The authors acknowledge Ann Marie Savage and the nurses of the Family Birth Center at Kapiolani Medical Center for Women and Children for their valuable support, as well as the patients who donated their time and samples.

\section{References}

Andraweera PH, Dekker GA \& Roberts CT 2012 The vascular endothelial growth factor family in adverse pregnancy outcomes. Human Reproduction Update 18 436-457. (doi:10.1093/humupd/dms011)

Barker DJ 2006 Adult consequences of fetal growth restriction. Clinical Obstetrics and Gynecology 49 270-283. (doi:10.1097/00003081200606000-00009) 
Barker DJ, Thornburg KL, Osmond C, Kajantie E \& Eriksson JG 2010 The surface area of the placenta and hypertension in the offspring in later life. International Journal of Developmental Biology 54 525-530. (doi:10.1387/ijdb.082760db)

Bartsch O, Bartlick B \& Ivell R 2001 Relaxin signalling links tyrosine phosphorylation to phosphodiesterase and adenylyl cyclase activity. Molecular Human Reproduction 7 799-809. (doi:10.1093/ molehr/7.9.799)

Bathgate RA, Halls ML, van der Westhuizen ET, Callander GE, Kocan M \& Summers RJ 2013 Relaxin family peptides and their receptors. Physiological Reviews 93 405-480. (doi:10.1152/ physrev.00001.2012)

Catalano PM, Presley L, Minium J \& Hauguel-de Mouzon S 2009 Fetuses of obese mothers develop insulin resistance in utero. Diabetes Care 32 1076-1080. (doi:10.2337/dc08-2077)

Clark DE, Smith SK, Sharkey AM \& Charnock-Jones DS 1996 Localization of VEGF and expression of its receptors flt and KDR in human placenta throughout pregnancy. Human Reproduction 11 1090-1098. (doi:10.1093/oxfordjournals.humrep.a019303)

Clifton VL 2005 Sexually dimorphic effects of maternal asthma during pregnancy on placental glucocorticoid metabolism and fetal growth. Cell and Tissue Research 322 63-71. (doi:10.1007/s00441-0051117-5)

Conrad KP 2011 Emerging role of relaxin in the maternal adaptations to normal pregnancy: implications for preeclampsia. Seminars in Nephrology 31 15-32.

Demetriou C, Abu-Amero S, Thomas AC, Ishida M, Aggarwal R, Al-Olabi L, Leon LJ, Stafford JL, Syngelaki A, Peebles D et al. 2014 Paternally expressed, imprinted insulin-like growth factor- 2 in chorionic villi correlates significantly with birth weight. PLOS ONE 9 e85454. (doi:10.1371/journal.pone.0085454)

Eder K, Baffy N, Falus A \& Fulop AK 2009 The major inflammatory mediator interleukin-6 and obesity. Inflammation Research 58 727-736. (doi:10.1007/s00011-009-0060-4)

Ferraro ZM, Qiu Q, Gruslin A \& Adamo KB 2012 Characterization of the insulin-like growth factor axis in term pregnancies complicated by maternal obesity. Human Reproduction 27 2467-2475. (doi:10.1093/ humrep/des189)

Fiore C, Bailey D, Conlon N, Wu X, Martin N, Fiorentino M, Finn S, Fall K, Andersson S, Andren O et al 2012 Utility of multispectral imaging in automated quantitative scoring of immunohistochemistry. Journal of Clinical Pathology 65 496-502. (doi:10.1136/jclinpath-2012-200734)

Fisher SC, Kim SY, Sharma AJ, Rochat R \& Morrow B 2013 Is obesity still increasing among pregnant women? Prepregnancy obesity trends in 20 states, 2003-2009. Preventive Medicine 56 372-378. (doi:10.1016/j. ypmed.2013.02.015)

Fowden AL 2003 The insulin-like growth factors and feto-placental growth. Placenta 24 803-812. (doi:10.1016/S0143-4004(03)00080-8)

Garibay-Tupas JL, Maaskant RA, Greenwood FC \& Bryant-Greenwood GD 1995 Characteristics of the binding of 32P-labelled human relaxins to the human fetal membranes. Journal of Endocrinology 145 441-448. (doi:10.1677/joe.0.1450441)

Gonzalez-Bulnes A, Torres-Rovira L, Astiz S, Ovilo C, Sanchez-Sanchez R, Gomez-Fidalgo E, Perez-Solana M, Martin-Lluch M, Garcia-Contreras C \& Vazquez-Gomez M 2015 Fetal sex modulates developmental response to maternal malnutrition. PLOS ONE 10 e0142158. (doi:10.1371/journal. pone.0142158)

Halls ML, Hewitson TD, Moore XL, Du XJ, Bathgate RA \& Summers RJ 2009 Relaxin activates multiple cAMP signaling pathway profiles in different target cells. Annals of the New York Academy of Sciences $\mathbf{1 1 6 0}$ 108-111. (doi:10.1111/j.1749-6632.2008.03814.x)

Hansell DJ, Bryant-Greenwood GD \& Greenwood FC 1991 Expression of the human relaxin $\mathrm{H} 1$ gene in the decidua, trophoblast, and prostate. Journal of Clinical Endocrinology and Metabolism 72 899-904. (doi:10.1210/jcem-72-4-899)

Hayes ES 2004 Biology of primate relaxin: a paracrine signal in early pregnancy? Reproductive Biology and Endocrinology 236. (doi:10.1186/1477-7827-2-36)

Higgins L, Greenwood SL, Wareing M, Sibley CP \& Mills TA 2011 Obesity and the placenta: a consideration of nutrient exchange mechanisms in relation to aberrant fetal growth. Placenta 32 1-7. (doi:10.1016/j. placenta.2010.09.019)
Hisaw FL 1926 Experimental relaxation of the pubic ligament of the guinea pig. Experimental Biology and Medicine 23 661-663. (doi:10.3181/00379727-23-3107)

Hoyo C, Fortner K, Murtha AP, Schildkraut JM, Soubry A, DemarkWahnefried W, Jirtle RL, Kurtzberg J, Forman MR, Overcash F et al. 2012 Association of cord blood methylation fractions at imprinted insulin-like growth factor 2 (IGF2), plasma IGF2, and birth weight. Cancer Causes and Control 23 635-645. (doi:10.1007/s10552-012-9932-y)

Hsu SY, Nakabayashi K, Nishi S, Kumagai J, Kudo M, Sherwood OD \& Hsueh AJ 2002 Activation of orphan receptors by the hormone relaxin. Science 295 671-674. (doi:10.1126/science.1065654)

Jelinic M, Leo CH, Post Uiterweer ED, Sandow SL, Gooi JH, Wlodek ME, Conrad KP, Parkington H, Tare M \& Parry LJ 2014 Localization of relaxin receptors in arteries and veins, and region-specific increases in compliance and bradykinin-mediated relaxation after in vivo serelaxin treatment. FASEB Journal 28 275-287. (doi:10.1096/fj.13-233429)

Johnson MR, Abdalla H, Allman AC, Wren ME, Kirkland A \& Lightman SL 1991 Relaxin levels in ovum donation pregnancies. Fertility and Sterility 56 59-61. (doi:10.1016/S0015-0282(16)54416-1)

Kappil MA, Green BB, Armstrong DA, Sharp AJ, Lambertini L, Marsit CJ \& Chen J 2015 Placental expression profile of imprinted genes impacts birth weight. Epigenetics 10 842-849. (doi:10.1080/15592294.2015.1 073881)

Langer O, Yogev Y, Most O \& Xenakis EM 2005 Gestational diabetes: the consequences of not treating. American Journal of Obstetrics and Gynecology 192 989-997. (doi:10.1016/j.ajog.2004.11.039)

Lowndes K, Amano A, Yamamoto SY \& Bryant-Greenwood GD 2006 The human relaxin receptor (LGR7): expression in the fetal membranes and placenta. Placenta 27 610-618. (doi:10.1016/j. placenta.2005.07.011)

Ma S, Sang Q, Lanciego JL \& Gundlach AL 2009 Localization of relaxin-3 in brain of Macaca fascicularis: identification of a nucleus incertus in primate. Journal of Comparative Neurology 517 856-872. (doi:10.1002/ cne.22197)

Magann EF, Doherty DA, Sandlin AT, Chauhan SP \& Morrison JC 2013 The effects of an increasing gradient of maternal obesity on pregnancy outcomes. Australian and New Zealand Journal of Obstetrics and Gynaecology 53 250-257. (doi:10.1111/ajo.12047)

Mazella J, Tang M \& Tseng L 2004 Disparate effects of relaxin and TGFbeta1: relaxin increases, but TGFbeta 1 inhibits, the relaxin receptor and the production of IGFBP-1 in human endometrial stromal/decidual cells. Human Reproduction 19 1513-1518. (doi:10.1093/humrep/ deh274)

McMinn J, Wei M, Schupf N, Cusmai J, Johnson EB, Smith AC, Weksberg R, Thaker HM \& Tycko B 2006 Unbalanced placental expression of imprinted genes in human intrauterine growth restriction. Placenta $\mathbf{2 7}$ 540-549. (doi:10.1016/j.placenta.2005.07.004)

McMurtry JP, Floersheim GL \& Bryant-Greenwood GD 1980 Characterization of the binding of 125I-labelled succinylated porcine relaxin to human and mouse fibroblasts. Journal of Reproduction and Fertility 58 43-49. (doi:10.1530/jrf.0.0580043)

Meller M, Vadachkoria S, Luthy DA \& Williams MA 2005 Evaluation of housekeeping genes in placental comparative expression studies. Placenta 26 601-607. (doi:10.1016/j.placenta.2004.09.009)

Millar LK, Reiny R, Yamamoto SY, Okazaki K, Webster L \& BryantGreenwood GD 2003 Relaxin causes proliferation of human amniotic epithelium by stimulation of insulin-like growth factor-II. American Journal of Obstetrics and Gynecology 188 234-241. (doi:10.1067/ mob.2003.80)

Novak J, Parry LJ, Matthews JE, Kerchner LJ, Indovina K, Hanley-Yanez K, Doty KD, Debrah DO, Shroff SG \& Conrad KP 2006 Evidence for local relaxin ligand-receptor expression and function in arteries. FASEB Journal 20 2352-2362. (doi:10.1096/fj.06-6263com)

Rochester D, Jain A, Polotsky AJ, Polotsky H, Gibbs K, Isaac B, Zeitlian G, Hickmon C, Feng S \& Santoro N 2009 Partial recovery of luteal function after bariatric surgery in obese women. Fertility and Sterility $\mathbf{9 2}$ 1410-1415. (doi:10.1016/j.fertnstert.2008.08.025)

Roland MC, Friis CM, Godang K, Bollerslev J, Haugen G \& Henriksen T 2014 Maternal factors associated with fetal growth and birthweight are independent determinants of placental weight and exhibit differential effects by fetal sex. PLOS ONE 9 e87303. (doi:10.1371/journal. pone.0087303) 
Rosenfeld CS 2015 Sex-specific placental responses in fetal development. Endocrinology 156 3422-3434. (doi:10.1210/en.2015-1227)

Sarwar M, Samuel CS, Bathgate RA, Stewart DR \& Summers RJ 2015 Serelaxin-mediated signal transduction in human vascular cells: bellshaped concentration-response curves reflect differential coupling to $C$ proteins. British Journal of Pharmacology 172 1005-1019. (doi:10.1111/ bph.12964)

Savvidou MD, Yu CK, Harland LC, Hingorani AD \& Nicolaides KH 2006 Maternal serum concentration of soluble fms-like tyrosine kinase 1 and vascular endothelial growth factor in women with abnormal uterine artery Doppler and in those with fetal growth restriction. American Journal of Obstetrics and Gynecology 195 1668-1673. (doi:10.1016/j. ajog.2006.03.065)

Shuman C, Beckwith JB, Smith AC \& Weksberg R 1993-2016 BeckwithWiedemann syndrome. In GeneReviews. Seattle (WA), USA: University of Washington.

Simchen MJ, Weisz B, Zilberberg E, Morag I, Weissmann-Brenner A, Sivan E \& Dulitzki M 2014 Male disadvantage for neonatal complications of term infants, especially in small-for-gestational age neonates. Journal of Maternal-Fetal and Neonatal Medicine 27 839-843. (doi:10.3109/1476 7058.2013.845658)

Smith CJ, Ryckman KK, Barnabei VM, Howard BV, Isasi CR, Sarto GE, Tom SE, Van Horn LV, Wallace RB \& Robinson JG 2016 The impact of birth weight on cardiovascular disease risk in the Women's Health Initiative. Nutrition, Metabolism, and Cardiovascular Diseases 26 239-245. (doi:10.1016/j.numecd.2015.10.015)

Stack EC, Wang C, Roman KA \& Hoyt CC 2014 Multiplexed immunohistochemistry, imaging, and quantitation: a review, with an assessment of tyramide signal amplification, multispectral imaging and multiplex analysis. Methods 70 46-58. (doi:10.1016/j. ymeth.2014.08.016)

Tashima LS, Yamamoto SY, Yasuda M, Millar LK \& Bryant-Greenwood GD 2002 Decidual relaxins: gene and protein up-regulation in preterm premature rupture of the membranes by complementary DNA arrays and quantitative immunocytochemistry. American Journal of Obstetrics and Gynecology 187 785-797. (doi:10.1067/mob.2002.125763)

Taylor CR \& Levenson RM 2006 Quantification of immunohistochemistry - issues concerning methods utility and semiquantitative assessment Histopathology 49 411-424. (doi:10.1111/j.1365-2559.2006.02513.x)

Tsai PJ, Davis J \& Bryant-Greenwood G 2015a Systemic and placental leptin and its receptors in pregnancies associated with obesity. Reproductive Sciences 22 189-197. (doi:10.1177/1933719114537718)
Tsai PJ, Davis J, Thompson K \& Bryant-Greenwood G 2015b Visfatin/ Nampt and SIRT1: roles in postterm delivery in pregnancies associated with obesity. Reproductive Sciences 22 1028-1036. (doi:10.1177/1933719115570908)

Unemori EN, Erikson ME, Rocco SE, Sutherland KM, Parsell DA, Mak J \& Grove BH 1999 Relaxin stimulates expression of vascular endothelial growth factor in normal human endometrial cells in vitro and is associated with menometrorrhagia in women. Human Reproduction 14 800-806. (doi:10.1093/humrep/14.3.800)

Unemori EN, Lewis M, Constant J, Arnold G, Grove BH, Normand J, Deshpande U, Salles A, Pickford LB, Erikson ME et al. 2000 Relaxin induces vascular endothelial growth factor expression and angiogenesis selectively at wound sites. Wound Repair and Regeneration 8 361-370. (doi:10.1111/j.1524-475X.2000.00361.x)

Unterscheider J, O'Donoghue K, Daly S, Geary MP, Kennelly MM, McAuliffe FM, Hunter A, Morrison JJ, Burke G, Dicker P et al. 2014 Fetal growth restriction and the risk of perinatal mortality-case studies from the multicentre PORTO study. BMC Pregnancy and Childbirth $\mathbf{1 4}$ 63. (doi:10.1186/1471-2393-14-63)

van Drongelen J, van Koppen A, Pertijs J, Gooi JH, Parry LJ, Sweep FC, Lotgering FK, Smits P \& Spaanderman ME 2012 Impaired vascular responses to relaxin in diet-induced overweight female rats. Journal of Applied Physiology 112 962-969. (doi:10.1152/ japplphysiol.00470.2011)

Wallace ME, Mendola P, Kim SS, Epps N, Chen Z, Smarr M, Hinkle SN, Zhu Y \& Grantz KL 2016 Racial/ethnic differences in preterm perinatal outcomes. American Journal of Obstetrics and Gynecology 216 306.e1-306.e12. (doi:10.1016/j.ajog.2016.11.1026)

Weiss G, O'Byrne EM \& Steinetz BG 1976 Relaxin: a product of the human corpus luteum of pregnancy. Science 194 948-949. (doi:10.1126/ science.982052)

Received 22 January 2017

First decision 8 March 2017

Revised manuscript received 4 April 2017

Accepted 2 May 2017 